\title{
Psychologie und Pandemie
}

\section{Die Auswirkungen des Corona-Virus auf den Einzelnen und auf die Gesellschaft}

Korrespondenzadresse

Prof. Dr. Dr. Manfred Spitzer

Universität Ulm

Leimgrubenweg 12-14

87054 Ulm
Bibliografie

DOI https://doi.org/10.1055/a-1094-9461

Nervenheilkunde 2020; 39: 274-283

(c) Georg Thieme Verlag KG Stuttgart · New York

ISSN 0722-1541
Noch nie habe ich ein Editorial für die Nervenheilkunde in dem Wissen geschrieben, dass es veraltet sein wird, wenn es erscheint. Aber inmitten einer Pandemie mit noch immer exponentiellem Verlauf ist auch der späteste weitgehende Redaktionsschluss (6.4.2020) um Wochen zu früh, und die weltweiten 1,2 Millionen infizierten Menschen sowie 64000 Toten werden längst (noch vergleichsweise „harmlose“) Vergangenheit sein. Warum schreibe ich trotzdem?

Eines war klar: Ich kann nicht „nicht“ über die Corona-Pandemie schreiben, denn einerseits durchleben wir gerade eine globale Pandemie, „für die es bislang kein Drehbuch gibt“ (so der deutsche Finanzminister Olaf Scholz), und andererseits leidet der überwiegende Teil der Menschen hierzulande (noch) nicht unter dem Virus, sondern unter sich selbst: Ängste und Ungewissheit, soziale Distanz und Einsamkeit, gleichzeitig mit sozialem Druck und Überflutung durch immer bedrohlichere Nachrichten, FakeNews und Verschwörungstheorien und Politiker (glücklicherweise vor allem ausländische), die gar nichts zu begreifen scheinen und keine oder die falschen Maßnahmen beschließen oder sich gar selbst widersprechen. Bei alldem geht es um uns Menschen und erst in zweiter Linie um das Virus. Deswegen ist gerade jetzt neben dem Virologen und Epidemiologen auch der Psychiater herausgefordert.

In lebenspraktischer Hinsicht hat das in den letzten Wochen sehr viel Arbeit gemacht und Selbstbeherrschung gekostet. Chefvisite mit Mundschutz und ohne Handschlag hätte ich mir bis vor wenigen Wochen weder vorstellen können, noch wollen, aber es muss nun sein (und es zeigte sich, dass nicht nur Minister Mühe haben, den Handschlag zu unterdrücken). Das gesamte Universitätsklinikum Ulm (UKU) befindet sich seit Wochen im Corona-Notfall-Modus: Verschiedene Teams planen die Versorgung von zu erwartenden künftigen Kranken. Ich selbst bin seit 3 Wochen Mitglied eines Umsetzungsteams, das täglich direkt im Anschluss an die KrisenTask-Force zusammenkommt und sich überlegt, wie man praktisch umsetzt, was zuvor von Klinikleitung, Anästhesie, Chirurgie und Innerer Medizin prinzipiell beschlossen wurde.

Seit dem 16. März erfinden wir in der Psychiatrischen Klinik in Ulm fast täglich die Psychiatrie neu, um unter sich ständig verändernden Bedingungen das zu tun, was wir immer tun: unsere Patienten weiter wie bisher so gut wie möglich zu versorgen. Diese Veränderungen müssen sein, denn die von Kanzlerin Merkel am Donnerstag den 12.3. zu Recht geforderte und in ihrer Rede an die Nation am 18.3. nochmals eindringlich betonte sozialen Distanz passt nicht zu Gruppentherapien, die in den Bereichen Sport, Musik, Kunst, manuelles Arbeiten mit verschiedenen Materialien und Zielen sowie im psychotherapeutischen Bereich zum Standard der Versorgung stationärer Patienten gehören. Seit Immanuel Kant [20], dem Philosophen der Aufklärung, wissen wir, dass psychische Krankheit mit einem Verlust des sensus communis einhergeht, weswegen die „therapeutische Gemeinschaft“ das Rückgrat unseres Tuns darstellt.

Ganz allgemein und weder nur für die Psychiatrie noch nur für Ulm gilt: In einigen Monaten werden wir wissen, wie Deutschland und sein gesamtes gesundheitliches Versorgungssystem mit der schwersten Belastungsprobe fertig geworden sein werden, die es - um mit der Kanzlerin zu reden - seit dem Zweiten Weltkrieg gab. Eine solche Situation haben die meisten Menschen noch nicht erlebt. Was bedeutet dies alles für jeden einzelnen?

Es geht im Folgenden also weder um das Virus SARS-CoV-2 noch die von ihm verursachte Krankheit Covid-19, sondern um uns - unser Erleben, Fühlen, Bewerten, Entscheiden und Handeln in Zeiten von Krisen - als Einzelne, als Gruppe und als Gesellschaft. Wie sich mittlerweile herumspricht, sind unsere Verhaltensweisen und Reaktionen auf das Virus für die Bewältigung der Krise mindestens so wichtig wie das Wissen über das Virus selbst. Was also wissen wir? - Nicht erst seit Corona durchaus eine ganze Menge.

\section{Risikobewertung und exponentielles Wachstum trifft auf komplexe Systeme}

Menschen tun sich schwer dabei, die Bedeutung kleiner Risiken abzuschätzen [23]. Diese Unfähigkeit liegt letztlich dem Geschäftsmodell von Lotterien und Versicherungen zugrunde. Wir Menschen verstehen einfache Zusammenhänge: Wenn es regnet, wird die Erde nass - je mehr, desto mehr. Es gibt aber auch andere Zusammenhänge, z. B. quadratische wie den umgekehrt-U-förmigen Zusammenhang zwischen Körpertemperatur und Gesundheit: ein paar Grad zu tiefe oder zu hohe Körpertemperatur, und es geht uns sehr schlecht; noch ein paar Grad mehr und wir sind tot. Das verstehen die meisten gerade noch. Exponentielle Zusammenhänge verstehen die meisten Menschen nicht: Stellen Sie sich bitte einen See vor, auf dem Seerosen wachsen, die sich täglich aufs Doppelte vermehren: Am ersten Tag eine, am zweiten Tag zwei, am drit- 


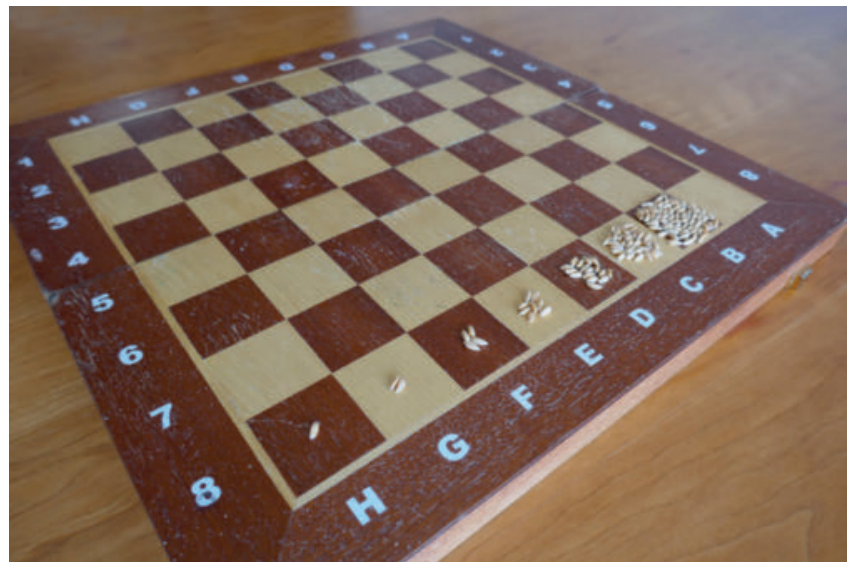

- Abb. 1 Weizenkörner auf einem Schachbrett. Beginnt man mit 1 Korn auf dem ersten Feld und verdoppelt die Zahl auf dem nächsten Feld usw. nimmt die Zahl der Körner exponentiell zu und schon auf das achte Feld passen die Körner nicht mehr: 1, 2, 4, 8, 16 etc., die Zahl der Körner folgt also der Formel $2^{(n-1)}$, wobei n die „Nummer des Feldes ist. Folglich befinden sich auf dem ersten Feld $2^{0=1}$, auf dem zweiten Feld $2^{1=2}$, auf dem dritten Feld $2^{2=4}$ und auf dem 64. Feld $2^{63}$ Körner (Quelle: @Autor)

ten Tag vier usw. Am dreißigsten Tag ist der ganze See bedeckt mit Seerosen. Wie bedeckt war der See einen Tag zuvor?
(A) $99 \%$,
(B) $95 \%$,
(C) $90 \%$,
(D) $75 \%$,
(E) $50 \%$

Die meisten Menschen vermuten hier eine Lösung irgendwo im mittleren Bereich (also B bis D), richtig ist jedoch E: Am Tag bevor der See voller Seerosen ist, war er erst halbvoll! Man spricht von exponentiellem Wachstum. Betrachten wir noch ein sehr bekanntes Beispiel: Wenn man auf das erste Kästchen eines Schachbretts ein Weizenkorn legt, auf das zweite 2 und auf das nächste 4 und so weiter, auf wieviel Weizen kommt man am Ende? Die Antwort lautet $2^{63}$, d. h. 9223372036854775808 Körner. Da die Tausendkornmasse von Weizen bei etwa $50 \mathrm{~g}$ (40 bis 65 Gramm) liegt, ergibt das 461168601842 Tonnen Weizen, oder etwa 5555 Tonnen für jeden der 83 Millionen Bundesbürger.

Weil wir große Schwierigkeiten beim Verstehen exponentiellen Wachstums haben, verstehen wir die gegenwärtige Krise so wenig. „Seit es die Corona-Krise gibt, sind doch erst 27 Menschen am Virus gestorben - was soll die ganze Panikmache? Im Straßenverkehr sind im gleichen Zeitraum viel mehr Menschen gestorben. “ - So oder so ähnlich dachten noch vor wenigen Wochen viele. Aber der Verkehr wächst nicht exponentiell wie die Seerosen und die Weizenkörner - und die Coronavirus-Fälle und -Toten!

Wenn man den Verlauf der Pandemie in Ländern wie Großbritannien, Schweden oder den USA betrachtet, wird sofort klar, wie todbringend mathematische Unkenntnis sein kann. Am 20. März 2020 gab es in den USA noch 14631 infizierte Corona-Fälle. In Deutschland waren es zum gleichen Zeitpunkt schon 18361 Fälle und in Großbritannien erst 3297. Genau eine Woche später (am 27.3.) lagen die Zahlen wie folgt: USA: 100717 , Deutschland 50871 und Großbritannien 14734. Die Zahl der Fälle war damit innerhalb einer Woche in den USA etwa auf das 7-Fache, in Großbritannien auf das 4,5-Fache und in Deutschland auf das 2,5-Fache gestiegen ( $\triangleright$ Abb. 2).

Der britische Premierminister Boris Johnson folgte seinem Berater viel zu lange, der gemeint hatte, man müsse nur abwarten,

\section{HINTERGRUNDWISSEN}

\section{Corona (Stand: 6.4.2020)}

Corona-Viren kommen in Fledermäusen und Pangolins vor. Wahrscheinlich schon im November 2019, vielleicht auch schon Ende September 2019 [16], kam es zu einem Übergang des Virus, möglicherweise von Fledermäusen über Pangolins auf den Menschen [29], vermutlich dadurch, dass es in China Märkte für alle möglichen (noch lebendigen) Lebewesen gibt, die zum Verzehr gekauft werden (weswegen mittlerweile ein komplettes Verbot des Verkaufs solcher „Wildtiere“ zum Verzehr in ganz China diskutiert wird [26, 27]). Machenschaften in einem chinesischen Hochsicherheitslabor haben das Virus nicht hervorgebracht, wie dessen genetische Analyse zeigt [2].

Dann verbreitete sich das Virus bekanntermaßen zunächst in der Stadt Wuhan in der chinesischen Provinz Hubei. Der erste Fall von Covid-19 wurde im Dezember 2019 von dem 33-jährigen Augenarzt Li Wenliang beschrieben, der über Weibo, das chinesische Analogon von Twitter, seine Kollegen warnen wollte. Dafür wurde er von der Polizei verprügelt und für einige Tage inhaftiert. Erst im Januar schwenkte die chinesische Regierung ihre Strategie von „Vertuschung“ auf „rigorose Maßnahmen zur Eindämmung“ um: Am 23. Januar 2020 wurden in Wuhan und ein Tag später in 14 weiteren Städten drakonische Maßnahmen zur Abriegelung eingeführt, die in der Folge die Verbreitung des Virus eindämmten, sodass es am 18. März 2020 erstmals keinen einzigen Neuerkrankten in China gab. Bereits am 7. Februar ist Wenliang, 33-jährig, verstorben und hinterließ Kind und Frau, die im Juli das zweite Kind erwartet.

Wichtige Pandemie-Parameter sind für das Virus SARS-CoV-2 bis heute nicht geklärt. Die Rate der Todesfälle (Letalität) beispielsweise schwankt zwischen 1 von Tausend bis 1 von 30. Das Risiko hängt von Alter, Geschlecht, Gesundheitszustand und der medizinischen Versorgung ab, die man erhält. In Großbritannien lag die Letalität an Covid-19 bei rund 9\%, in Italien bei fast $12 \%$ und in Deutschland bei nur gut $1 \%$. Die Letalität einer Erkrankung bezieht sich ja grundsätzlich auf eine bestimmte Gemeinschaft bzw. einer ganzen Gesellschaft. Besteht eine Altersabhängigkeit des Erkrankungsrisikos, dann wirkt sich das auf die Letalität entsprechend aus. So wurde berechnet, dass die Letalität in Nigeria mit seiner recht jungen Bevölkerung nicht einmal halb so groß sein dürfte wie die in Italien. Schließlich ist noch die Frage ungeklärt, wie viele Infizierte mit leichten oder keinen Symptomen bei diesen Berechnungen übersehen werden. Wenn es viele sind, wird die Letalität aller mit SARS-CoV-2 Infizierten viel niedriger sein als die Letalität von Covid-19, also von an der Krankheit leidenden Menschen. Wir wissen, dass Großbritannien nur schwerkranke Menschen testet und damit viele leichte oder symptomlose Fälle nicht berücksichtigt, Südkorea und Deutschland hingegen viele Tests durchgeführt haben. Daher ist die Letalität in diesen Ländern geringer [13]. Die Maßnahmen der sozialen Distanzierung sind schon seit über hundert Jahren bekannt und nachweislich wirksam [11]. 


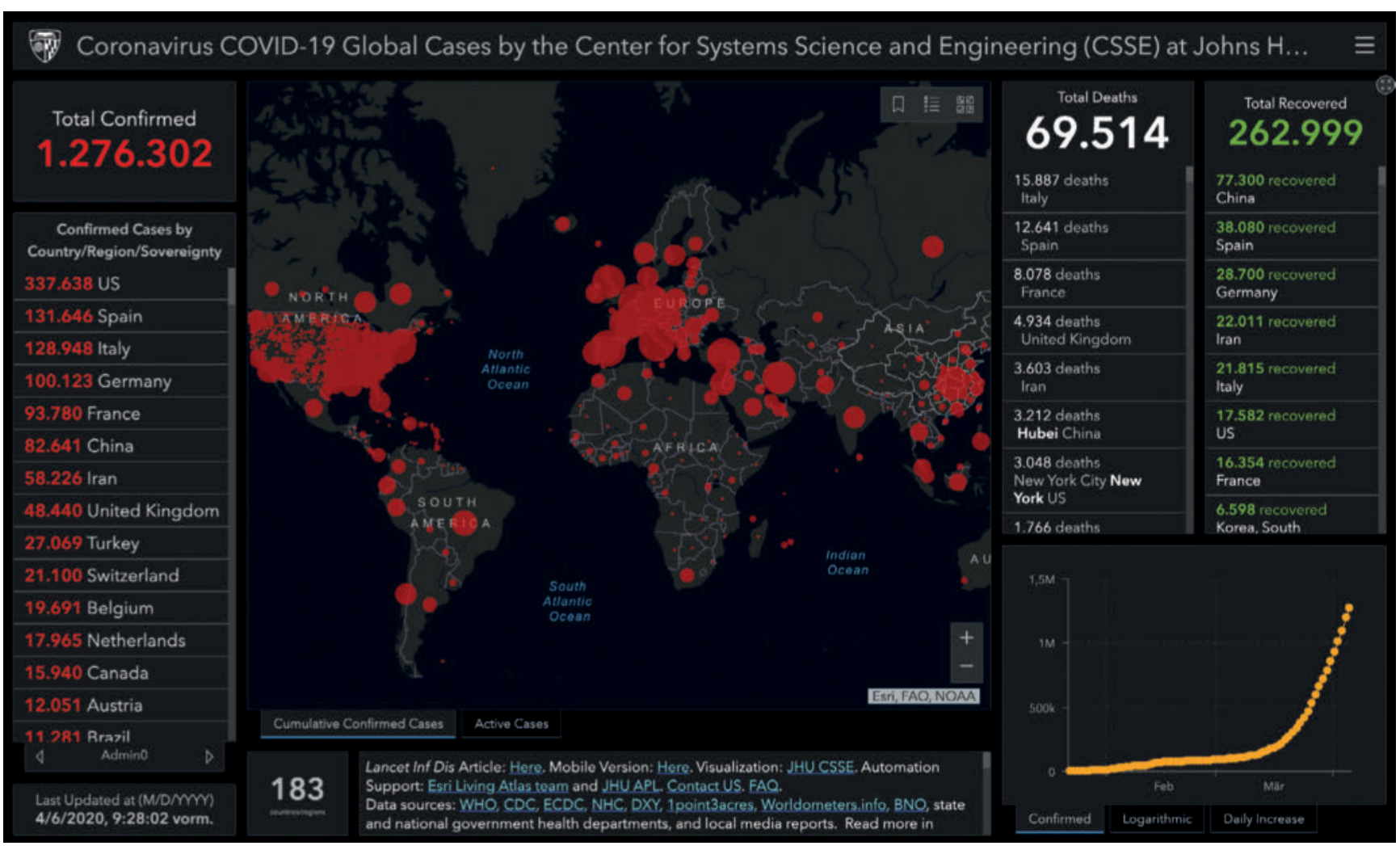

- Abb. 2 Das Online-Dashboard der Johns-Hopkins-Universität zählt mit über einer Milliarde Clicks wahrscheinlich zu den meist angesehenen Webseiten [31]. Man sieht hier die Daten aus 183 Ländern zu Infektionen (1276302 Fälle), Toten (69514) und wieder Genesenden (262999). Die Daten werden mehrfach täglich aktualisiert, und man kann sich interaktiv über einzelne Länder informieren. Wenn in dieser Arbeit keine anderen Quellen für Zahlen genannt werden, dann stammen sie aus dieser Quelle: https://gisanddata.maps.arcgis.com/apps/opsdashboard/index.html\#/ bda7594740fd40299423467b48e9ecf6 (abgerufen am 6.4.2020, um 9.38 Uhr).

bis Herdenimmunität ${ }^{1}$ bestehe, $d$. h. bis so viele Menschen die Infektion durchgemacht haben, dass die Ausbreitung des Virus sich von alleine verlangsamt. Wie britische Wissenschaftler vom Imperial College COVID-19 Response Team jedoch am 16. März online publizierten [9], würde diese Strategie zu mehr als einer halben Million vermeidbarer Todesfälle im Land führen. Am gleichen Tag änderte Johnson seine Strategie: „[Er] sagte, man solle alle unnötigen Reisen unterlassen und möglichst von zu Hause aus arbeiten. Kneipen, Clubs und andere soziale Räume sollten vermieden werden, und ganze Haushalte sollten sich selbst 14 Tage lang isolieren, falls ein Mitglied Symptome von Covid-19 entwickelt. Besonders für das Virus anfällige Menschen könnten bald gebeten werden, 12 Wochen zu Hause zu bleiben“2 [12]. Die Zahlen lassen vermuten, dass Johnson zu spät gehandelt hat.

1 Der Begriff Herdenimmunität stammt aus der Tiermedizin und beinhaltet auch, dass ein Teil der Tiere nicht überlebt. Bei diesem Begriff denkt der Laie jedoch vor allem an Immunität, die er auch gerne hätte, aber nicht an die Inkaufnahme des Todes eines oder vieler Menschen (d. h. auch seines eigenen).

2 „UK prime minister Boris Johnson said people should stop all unnecessary travel, and work from home if possible. He said pubs, clubs and other social spaces should be avoided and whole households should self-isolate for 14 days if any individual in it develops covid-19 symptoms. People who may be particularly vulnerable to the virus may soon be asked to stay home for 12 weeks.“
Für die USA wurde mit gleicher Methodik berechnet, dass es beim Nichtstun zu 2,2 Millionen zusätzlichen Toten kommen würde, was Herrn Trump offenbar wenig beeindruckte. Seine Art des Umgangs mit der Corona-Pandemie [7] dürfte als klassisches Beispiel von diesbezüglichem komplettem administrativem Versagen in die Geschichte eingehen, und es ist zu vermuten, dass dies zur Folge haben kann, dass er im Herbst nicht wiedergewählt werden wird. Einer von vielen Kommentaren hierzu lautete wie folgt: „Das Coronavirus könnte sich als eine Herausforderung erweisen, der Trump in keiner Weise gewachsen ist - und in der sich all seine bisherigen Rezepte und Show-Effekte als wirkungslos erweisen. Der Erreger Sars-CoV-2 schafft Fakten, die sich nicht länger verdrehen lassen. Trump kann die Virus-Krise längst nicht mehr kleinreden. Kein Tweet, keine Mauer kann die Ausbreitung eindämmen. Die Corona-Katastrophe macht vor nichts Halt [...] Das Virus könnte Trump das Amt kosten. - Wie konnte es so weit kommen? Der fahrlässige Umgang mit der Pandemie-Bedrohung in den USA gilt als Lehrbeispiel für katastrophal schlechtes Krisenmanagement“ [18].

Sowohl Trump als auch Johnson gehören dem konservativen Lager an. Ist das ein Zufall? Interessant ist im Hinblick auf diese Frage eine am 28. März als Vorabdruck erschienene Publikation von Wissenschaftlern um Christopher Adolph von der University of Washington in Seattle (USA). Sie konnten empirisch an einem großen Datensatz nachweisen, dass US-Bundesstaaten, die entweder einen republikanischen Gouverneur haben oder in denen Trump bei den Präsidentschaftswahlen im Jahr 2016 besser abgeschnitten 
hatte, Maßnahmen der körperlichen Distanzierung vergleichsweise mit geringerer Wahrscheinlichkeit und vor allem zu einem späteren Zeitpunkt eingeführt hatten als Staaten, die von Demokraten geführt oder dominiert werden ( $\triangleright$ Abb. $\mathbf{3}$ ).

Aus dem wesentlichen Absatz zum bisherigen Verlauf der Corona-Krise in den USA sei im Folgenden wörtlich zitiert: „Zu jedem beliebigen Zeitpunkt im Untersuchungszeitraum führten die republikanischen Gouverneure Maßnahmen der körperlichen Distanzierung mit 42,2\% (95\% KI: 13,5 bis 63,2) geringerer Wahrscheinlichkeit ein als ihre demokratischen Amtskollegen. Bei Konstanthaltung der anderen Variablen im jeweiligen Bundesstaat - einschließlich des Stimmenanteils von Trump bei den Wahlen im Jahr 2016 - verzögern Republikaner im Durchschnitt jede Maßnahme zur sozialen Distanzierung auf Bundesstaatsebene um 1,68 Tage (95\% KI: 1,57 bis 1,78). [...] Ein Bundesstaat, in dem der Trump-Stimmenanteil von 201675 \% erreichte, führte zu einem bestimmten Zeitpunkt mit 28,1 \% (95\% KI: 1,1 bis 49,3) geringerer Wahrscheinlichkeit eine zusätzliche Maßnahme [zur Eindämmung des Corona-Virus] ein als ein Bundesstaat, in dem die Unterstützung für Trump nur 25 \% erreichte. Die durchschnittliche Verzögerung betrug 0,99 Tage (95\% KI: 0,93 bis 1,07). In Staaten mit republikanischem Gouverneur und überwiegend Trump-Wählern (was zusammenhängt) war eine Verzögerung von 2,70 Tagen (95\% KI: 2,49 bis 2,88) zu verzeichnen. [...] Wenn sich die Zahl der Corona-Virus-Infektionen in einem Staat alle sieben Tage verdoppelt, erhöht sich die Spitzenbelastung der Fälle um 30,6\%. In einem Staat, in dem sich die Infektionen alle drei Tage verdoppeln, könnte das politische Überwiegen der republikanischen Partei die Spitzenzahl der Fälle um 86,6\% erhöhen“3 [1].

Nun ist die Spitzenbelastung in den USA noch nicht eingetreten. Wenn dies geschehen ist, wird man auch wissen, mit welcher Geschwindigkeit sich das Virus in dieser Zeit ausgebreitet hat. Dann wird jeder leicht berechnen können, in wie viele Tausenden oder Hunderttausenden Toten sich die Stimmen für Republikaner bzw. für Trump umrechnen lassen. Falsche Risikoeinschätzung und das Unverständnis für exponentielles Wachstum werden sich in den USA also besonders deutlich auswirken.

Als dritter Fall sei noch kurz Schweden diskutiert. Das Land belegte am 1. April 2020 (6 Uhr) mit 4435 Covid-19 positiven Fäl-

3

\footnotetext{
"Republican-leaning states are slower to adopt social distancing policies. At any given time within the study period, Republican governors were $42.2 \%$ (95\% Cl: 13.5 to 63.2 ) less likely to mandate social distancing than their Democratic counterparts. Holding constant other variables - including the 2016 Trump vote share - at their observed values in each state, on average, Republican governors delay each state-level social distancing measure by 1.68 days ( $95 \% \mathrm{Cl}$ : 1.57 to 1.78$)$. At the same time, holding constant the Governor's party affiliation, states with more Trump voters were less likely to adopt social distancing. A state at the 75th percentile of 2016 Trump vote share was $28.1 \%$ (95\% $\mathrm{Cl}: 1.1$ to 49.3 ) less likely to adopt an additional mandate at a given time when compared to a state at the 25th percentile of Trump support, which resulted in an average delay of 0.99 days $(95 \% \mathrm{Cl}: 0.93$ to 1.07). Republican governors often go together with Trump-voting electorates; on average, these states endured a combined partisan delay of 2.70 days ( $95 \% \mathrm{Cl}: 2.49$ to 2.88 ). Barring positive developments in the fight against COVID-19, the public health impact of this delay is likely to be massive: in a state where coronavirus infections are doubling every seven days, this would raise the peak caseload by $30.6 \%$. In a state where infections are doubling every three days, Republican partisanship might raise the peak level of cases by $86.6 \%$."
}

len im weltweiten Ranking (1. USA, 2. Italien, 3. Spanien, 4. China, 5. Deutschland, 6. Frankreich, 7. Iran, 8. Großbritannien) nur den Rangplatz 21, noch hinter Norwegen (mit 4641 Fällen) auf Platz 19. Die Anzahl der Todesfälle lag damals jedoch in Schweden mit 180 schon deutlich über der von Norwegen mit 39. Dennoch wurden in Schweden keine drastischen Maßnahmen verhängt, die Kneipen und Restaurants blieben offen und die Schulen und Kindergärten auch. Der dortige verantwortliche Epidemiologe Anders Tegnell riet der Regierung, einfach abzuwarten, bis genügend Schweden die Krankheit durchgemacht haben, wodurch dann Herdenimmunität für Schweden bestehe und sich das Problem erledigt haben würde. Dass er mit diesem Nichthandeln Tote produziere, muss er in Kauf genommen haben. Ob er der Regierung von den zu erwartenden Toten erzählt hat, weiß ich nicht, nach deren Verhalten - sie fangen jetzt - eine Woche vor Ostern - an, über ausbreitungshemmende Maßnahmen nachzudenken, scheint dies nicht der Fall gewesen zu sein.

Dass die Corona-Krise nicht nur unserem Unverständnis kleiner Wahrscheinlichkeiten und der Exponentialfunktion, sondern auch unserer unglaublich komplexen Gesellschaft geschuldet ist, wird jedem klar, der darüber nachdenkt. Unsere Gesellschaft weist schier unendlich viele funktionale Zusammenhänge physikalischer, chemischer, biologischer, psychologischer und sozialer Natur auf, die wir längst noch nicht alle kennen oder gar verstanden haben und die zugleich oft nicht linear sind. Wenn nun auf dieses äußerst komplexe System eine globale Pandemie mit exponentiellem Wachstum zukommt, dann werden ständig Konsequenzen real, mit denen niemand gerechnet hatte.

Ein vielleicht noch nicht sehr bekanntes Beispiel ist die Wettervorhersage, die durch die Corona-Pandemie schlechter geworden ist, genau genommen etwa so schlecht wie vor 10 Jahren. - Warum? Durch die Corona-Krise ist es zu einem dramatischen Einbruch von Wetterdaten gekommen. Ursache für die fehlenden Wetterdaten ist eine geringere Anzahl von Flugzeugen in der Luft, die während ihres Fluges wichtige Wetterdaten über den Ist-Zustand der Atmosphäre sammeln. Früher erfolgte die Messung von Temperatur, Luftfeuchtigkeit, Windgeschwindigkeiten und -richtungen in verschiedenen Höhen mit Wetterballons. Dann wurden diese durch die immer größer werdende Flotte der Verkehrsflugzeuge ersetzt, die ohnehin weitaus mehr Daten generieren und damit auch liefern können. Durch die Eindämmung der Corona-Pandemie ist der Luftverkehr fast zum Erliegen gekommen. Und deswegen sind jetzt Unwetter schwerer vorherzusagen. Damit erhöht sich das Risiko für Länder, die mehr Vorlauf brauchen, um sich auf Wetterkatastrophen vorzubereiten. Wettervorhersagen sind zwar noch immer so gut wie vor 10 Jahren, und wenn es im Wetterbericht heißt, es wird morgen die Sonne scheinen, dann wird es morgen nicht regnen. Aber sie sind dennoch unzuverlässiger, vor allem für Zeitpunkte, die weiter in der Zukunft liegen.

\section{Angst und Misstrauen, Denunziation und Verleugnung}

Nicht nur unser Denken wird durch die Corona-Pandemie auf die Probe gestellt, auch unsere Gefühle. In evolutionärer Hinsicht handelt es sich dabei um Veränderungen des Gehirns und seiner Leistungen, die viele Funktionen betreffen und Heuristiken (sofort ver- 


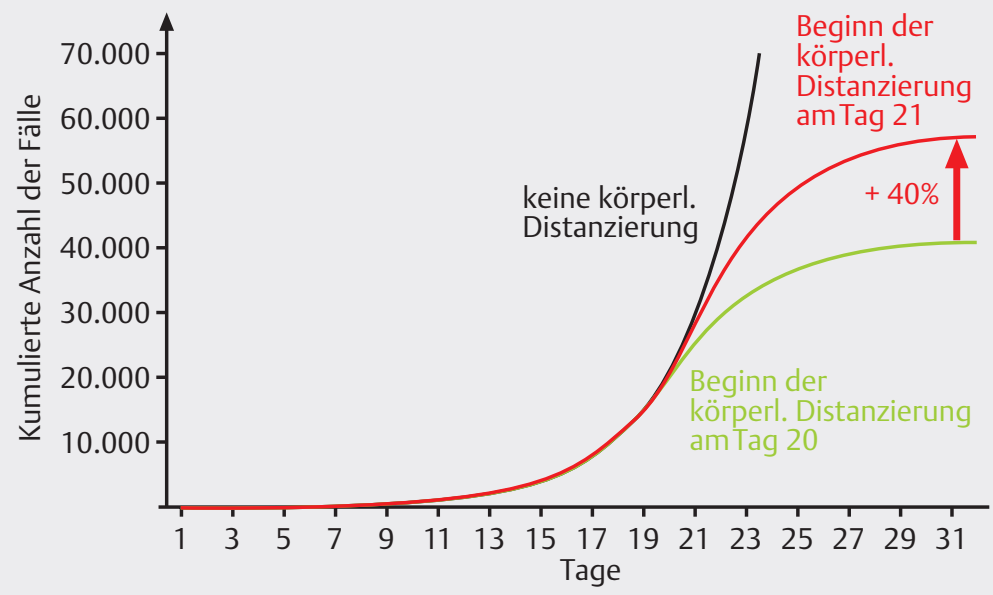

- Abb. 3 Effekt einer Maßnahme der sozialen Distanzierung in Abhängigkeit vom Zeitpunkt der Einführung: Ein Tag später resultiert in $40 \%$ mehr Fällen. Man sieht leicht ein, dass der Effekt von der Wachstumsrate, also der Steilheit der Kurve, abhängig ist (nach Daten aus [39]).

fügbare Richtschnüre) für rasches Handeln ohne langes Nachdenken (Deliberieren, Simulieren, Probehandeln) sorgen können. Wer aus irgendeinem Grunde Angst hat, denkt nicht lange nach, sondern tut das, was jetzt gerade notwendig ist, um aus der Situation wieder herauszukommen.

Wenn von der Psychologie der Corona-Pandemie die Rede ist, geht es meist um Angst und Einsamkeit. Meist wird darüber geredet, dass viele jetzt in Panik verfallen und sich dann aus diesem Grund unangemessen verhalten würden: Schlaflosigkeit, Sorgen, Grübeln und die resultierenden Hamsterkäufe oder ein generelles Misstrauen gegenüber allen fremden Menschen, die nur noch als Virus-Quelle wahrgenommen werden, sind die Folge. Diese Überreaktion vieler Einzelner schadet unserer Gesellschaft vor allem langfristig.

Denn wozu Misstrauen in der Pandemie führen kann, zeigen Fallbeispiele aus vielen Ländern. Wurde noch Mitte März fast schmunzelnd darüber berichtet, dass die Deutschen Mehl, Hefe und Toilettenpapier und die Franzosen Rotwein und Kondome in der Krise hamstern, so war die Nachricht, dass die Amerikaner vor allem Waffen kaufen, schon gar nicht mehr lustig. 2 Wochen später wurde dann berichtet, dass der Verkauf von Toilettenpapier in Deutschland tatsächlich um etwa ein Drittel zugenommen hat, der Verkauf von legalen Waffen zur Selbstverteidigung -hierzulande - hingegen auf das 3-Fache gestiegen ist.

In Frankreich gibt es 3,4 Millionen Ferienhäuser als Zweitwohnungen wohlhabender Bürger - mehr als in irgendeinem der Nachbarländer Frankreichs. Wie die New York Times am Sonntag, den 29.3.2020, berichtete, sind sehr viele Einwohner von Paris in den vergangenen Tagen auf's Land gefahren, um ihre Zeit in den Bergen oder am Meer zu verbringen. Auf der Atlantikinsel Noirmoutier liefen die neu Angekommenen dann gleich zum Strand und verhielten sich wie Urlauber, d. h. nicht ordnungsgemäß. Die Einheimischen fürchten sich vor ihnen und reagierten entsprechend: Bei Autos mit Pariser Nummernschild wurden die Reifen aufgeschlitzt [5]. Wenn wir schon darüber diskutieren, dass Grenzen zwischen EU-Ländern wie beispielsweise die deutsch-österreichischen Grenze im Grunde nicht geschlossen werden sollte, um wieviel mehr müssen wir uns dann um Landesgrenzen sorgen? Oder um den Zustand unse- rer Demokratie, in der kein Platz sein sollte für Denunzianten, auch nicht in sozialen Online-Netzwerken.

Aus meiner Sicht ist panische Überreaktion derzeit das kleinere Problem. Das genaue Gegenteil - Verleugnung und Kopf in den Sand - ist momentan weitaus gefährlicher. Denn es verhindert die jetzt nötigen Verhaltensänderungen, die unbedingt notwendig sind, wenn wir die Krise mit minimalen Verlusten - sprich: Todesfällen - überstehen wollen. Um es ganz klar zu sagen: Nach den derzeitigen Berechnungen werden die meisten Menschen am Corona-Virus versterben, weil ihre Mitmenschen zu sorglos mit der Gefahr umgegangen sind. So wie Brücken ja auch nur gefährlich sind, wenn man sich falsch verhält und von ihnen herunterspringt, ist das Corona-Virus nur gefährlich, wenn sich die Menschen nicht richtig verhalten. Corona-Partys in deutschen Großstädten oder am Strand in Florida sind gefährlicher als das Virus selbst. Wenn nach dem letzten Deutschlandtrend die Hälfte der Deutschen sich über Corona keine Sorgen machen, dann sind das definitiv $100 \%$ zu viele! Das muss sich ganz rasch ändern.

China hat seinen Bürgern während der Krise immer wieder eingehämmert: Ihr bringt andere um, wenn ihr Euch nicht an die Regeln haltet. Dieses Bewusstsein fehlt hier noch sehr deutlich, vor allem bei jüngeren Menschen, möglicherweise aus Sorglosigkeit oder eher Fahrlässigkeit, manche jedoch auch bewusst. Gehen Jugendliche und junge Erwachsene wirklich so gerne über Leichen? Und noch dazu über die von Oma und Opa? - Man muss sie vielleicht noch ein paarmal so deutlich fragen. Nichts nützen wird das bei denjenigen, die vom Corina-Virus zynisch als „Boomer-Remover" sprechen, d. h. als ein Agens, das die Generation der Baby-Boomer aus der Gesellschaft effektiv zu entfernen in der Lage ist [15].

\section{Körperlicher Abstand versus soziale Isolation versus Einsamkeit}

Die Erfahrungen aus Wuhan hatten gezeigt, dass körperlicher Abstand zwischen Personen (am besten 2 Meter) eine sehr gute Maßnahme darstellt, die Ausbreitung des Corona-Virus zu mindern. Im Englischen sprach man von „social distancing“, was man im Deut- 
schen übernahm, sodass auch die Kanzlerin in ihrer Rede an die Nation vom 18. März von einzuhaltender „sozialer Distanz“ bzw. „sozialer Distanzierung“ sprach. Man muss jedoch betonen, dass körperliche Distanz nicht das Gleiche ist wie soziale Isolation. Insofern sollte diese momentan wichtigste Maßnahme zur Verminderung der Todesfälle Maßnahme nicht „social distancing“, sondern „physical distancing“ genannt werden. Telefonieren beispielsweise bedeutet soziale Nähe bei körperlicher Distanz, und das wird während der Corona-Pandemie sehr wichtig. Weil dabei deutlich weniger Informationen durch das Netz fließen als beim Video-Chat, ist es zudem viel solidarischer, zu telefonieren, denn man blockiert mit dem Telefonieren weit weniger das Netz.

Auch die Weltgesundheitsorganisation spricht mittlerweile von „körperlicher“ und nicht mehr von „sozialer“ Distanz, die es einzuhalten gilt. In Singapur spricht man von „safe distancing“ und ein Psychologe der Stanford-University von „distant socializing.“ „Bleiben Sie zuhause und wenn Sie rausgehen, bleiben Sie körperlich entfernt voneinander." So sollte die lebenswichtige Nachricht formuliert werden, sagte ein Mitglied der Northwestern University, USA. „Aber um in dieser Zeit gesund zu bleiben, müssen die Leute aktiv nach Wegen suchen, sozial zu bleiben. Das ist etwas anderes als soziale Distanzierung“ " [2]. Laut der Nachrichtenagentur AFP unterlagt am 2. April mehr als die Hälfte der Weltbevölkerung (3,9 Milliarden Menschen) aufgrund der Corona-Pandemie Ausgangsbeschränkungen. In mehr als 90 Ländern waren demnach Ausgangssperren oder andere Maßnahmen in Kraft, die den Kontakt zwischen Menschen reduzieren sollten.

Das muss Nebenwirkungen haben. Denn die Vereinsamung durch Quarantäne ist eine reale und ernst zu nehmende Gefahr. Einsamkeit geht mit erhöhtem Stress einher, und wenn der chronisch wird, kommt es zu Bluthochdruck, Diabetes und verminderter Immunabwehr und damit zu mehr Herzinfarkten und Schlaganfällen sowie zu mehr Krebs und - gerade jetzt besonders wichtig - zu einem geschwächten Immunsystem und macht damit anfälliger für einen kritischen Verlauf der Corona-Infektionen [22]. Solange es weder nachweislich wirksame Medikamente oder Impfstoffe gibt, haben wir nichts anderes als unser eigenes Immunsystem zur Abwehr des Virus. Daher ist es auch sehr vernünftig, dass wir keine Ausgangssperre haben, sondern eine Reihe von Maßnahmen, die auf körperliche Distanz hinauslaufen: Fallbezogene Selbstisolation („Quarantäne“), Verbot öffentlicher Veranstaltungen (z. B. Fußballspiele), Kita und Schule geschlossen, Lockdown (wird unterschiedlich definiert und bezeichnet allgemein eine Verminderung vieler wirtschaftlicher Aktivitäten) und die Aufforderung zu körperlichem Abstandhalten von 2 Metern. Reisebeschränkungen gehören zwar auch dazu, bringen jedoch nach allem, was wir heute schon wissen, im Grunde sehr wenig bis gar nichts. Von den anderen genannten Maßnahmen ist der körperliche Abstand am wichtigsten.

Es ist daher schade, dass der Präsident des Landes, das schon länger die Liste der am meisten betroffen Länder anführt, weder das Tragen von Masken („das ist nichts für mich“) noch die körperliche Distanzierung vorlebt. Er wird mit seinem Motto „America first“" wohl kaum die Position der USA auf der Liste der Infektionszahlen gemeint haben.

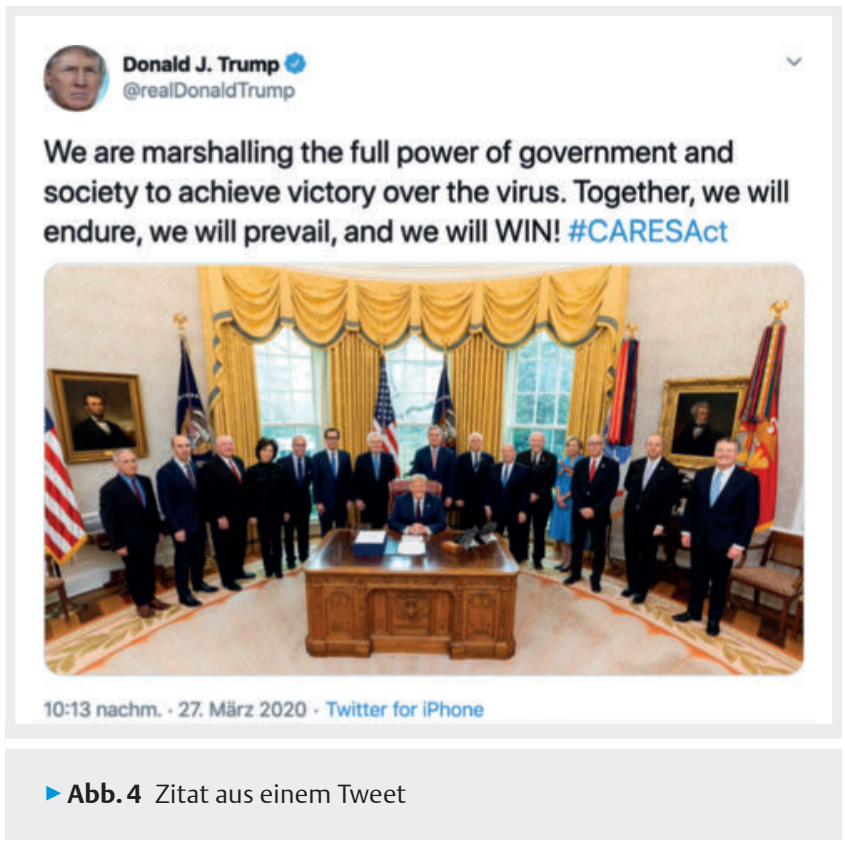

\section{Folgen}

Die Stadt New York war zum Zeitpunkt der Abfassung dieses Editorials zum weltweiten Zentrum der Pandemie geworden. Bereits am Donnerstag, den 26. März 2020, überstieg die Anzahl der Notrufe in der Stadt die Anzahl der Notrufe am 11. September 2001 [10]. Mittlerweile gibt es dort Kühllastwagen und Zelte für die täglich Hunderten von Corona-Toten sowie ein „Pop-up-Leichenschauhaus“. Pfarrer erteilen die Letzte Ölung per Video [28]. In den USA haben innerhalb der letzten beiden Märzwochen 10 Millionen Menschen Arbeitslosenhilfe beantragt und die Hälfte aller Amerikaner war Anfang April von Einkommensverlusten durch die Corona-Krise betroffen. Am 2. April hatte angesichts der steigenden Zahl von Corona-Toten die US-Katastrophenschutzbehörde das Verteidigungsministerium um 100000 Leichensäcke gebeten.

Ähnlich wie mit unserer Körpertemperatur, die ein Optimum zwischen den tödlichen Extremen „zu kalt“ und „zu warm“ hat, steht es auch mit unseren sozialen Beziehungen: Zuviel und zu wenig belasten kurzfristig und bringen uns langfristig um. Einsamkeit ist in den westlichen Industrienationen die Todesursache Nummer 1 und Stress durch fehlende Rückzugsmöglichkeiten (man spricht von „Dichtestress“) bewirkt Konflikte, die in Gewaltakten eskalieren können. In Wuhan steigen die Scheidungsraten und Psychologenverbände warnen vor steigendem Risiko häuslicher Gewalt. In Berlin ist die häusliche Gewalt um 10 \% gestiegen. In Deutschland gibt es ein Stadt-Land-Gefälle, weil man auf dem Land mehr Platz hat und bessere Möglichkeiten, rauszugehen.

Die Corona-Pandemie gefährdet unsere Demokratie. Sie erlaubt beispielsweise Despoten, die Versammlungsfreiheit einzuschränken oder sogar ganz aufzuheben, in Hongkong und Ungarn ebenso wie in China, Russland und Ländern in Südamerika. Die freiheitliche westliche Welt muss wachsam bleiben, jetzt und in der nahen Zukunft. Die Einschränkungen unserer Freiheit aufgrund der Corona-Pandemie werden nach heutigem Kenntnisstand definitiv nicht am 19. April enden, also dem Termin, bis zu dem die getroffenen Maßnahmen zunächst gelten sollten. Unsere Fähigkeit, sowohl mit 
Einsamkeit als auch mit Dichtestress umzugehen, wird auf eine harte Probe gestellt werden.

Machen wir uns keine Illusionen: Die Welt wird nach der Krise eine andere sein als davor. Wird sie eine bessere oder eine schlechtere sein? Das hängt - und es ist meiner Ansicht nach sehr wichtig, sich dies vor Augen zu führen - nicht vom Virus ab, sondern von uns ( $\triangleright$ Tab. 1).

Können Sie sich noch an die Meldungen des Robert-Koch-Instituts im vergangenen Sommer über die möglichen Tausende von Toten durch Feinstaubbelastung erinnern? Oder an die Fridays-for-Future-Bewegung und Greta Thunberg? Von beidem hört man jetzt gar nichts mehr, und der Grund ist nicht, dass wir das vergessen haben, sondern, dass die Forderungen erfüllt sind: $95 \%$ aller Flüge eingestellt, Ölförderung gedrosselt (weltweit), Wirtschaft gedrosselt (weltweit), bessere Luft in China und sauberes Wasser in Venedig. Ein winziges Viruspartikel, das uns Menschen eine Lungenkrankheit pandemischen Ausmaßes beschert hat, verschafft dem Erdball gerade eine Verschnaufpause. Die „Nebenwirkungen“ dieser globalen Rosskur für uns Menschen sind jedoch dramatisch und werden nicht nur viele Tote, sondern auch viel Leid (auch durch die wirtschaftlichen Einschnitte) mit sich bringen. Wir werden auch erleben, dass andere Patienten vernachlässigt werden, weil man sich nur noch um Covid-19 kümmern kann. Bei der Ebola-Epidemie in Afrika hat dieser indirekte Effekt zu einer Verdopplung der Ebola-Todesfälle geführt [17].

Greta Thunberg, die zwischenzeitlich wahrscheinlich auch an COVID-19 erkrankt war, sagte am 24. März 2020 etwas ähnliches: „Die Corona-Krise zeigt deutlich, dass unsere gegenwärtigen Gesellschaften nicht nachhaltig sind. Wenn ein Virus innerhalb weniger Wochen die gesamte Wirtschaft auslöschen und Gesellschaften zum Erliegen bringen kann, dann ist das ein Beweis dafür, dass unsere Gesellschaften nicht sehr widerstandsfähig sind. Es zeigt aber auch, dass wir dann, wenn wir uns in einer Notlage befinden, handeln und unser Verhalten rasch ändern können. Und solangeädwir Solidarität und gesunden Menschenverstand haben, werden wir jede Krise überstehen" ${ }^{4}$ (Thunberg, zit. nach [24]).

Die Krise hat schon jetzt nachweisbare positive Nebeneffekte: In Deutschland gibt es seit den einschränkenden Maßnahmen deutlich weniger Verkehrsunfälle, in Italien gibt es im Vergleich zum Vorjahr $64 \%$ weniger Straftaten und in Spanien ist die Kriminalität in der Corona-Krise um 50 \% zurückgegangen [6]. Die Deutsche Bahn ist seit der Corona-Krise pünktlicher, hieß es am 5.4. um 5.44Uhr ebenfalls auf Bild.de im Live-Ticker. Driver-by-Geburtstagspartys gibt es nicht nur in den USA, sondern auch bei uns.

Fassen wir zusammen: Die Corona-Krise wird nicht nur durch die Eigenheiten des Virus SARS-CoV-2 und der von ihm verursachten Krankheit Covid-19 bestimmt, sondern auch dadurch, was und wie Menschen in Krisenzeiten erleben, fühlen, bewerten, entscheiden und handeln. Über das Virus wissen wir noch längst nicht alles, weswegen eine Zusammenfassung wissenschaftlicher Erkenntnis-

4

\footnotetext{
"The corona crisis really shows that our current societies are unsustainable. If one virus can wipe out the entire economy in a matter of weeks and shut down societies, then that is a proof that our societies are not very resilient. It also shows that once we are in an emergency, we can act and we can change our behaviour quickly. And as long as we have solidarity and common sense, we will get through any crisis."
}

- Tab. 1 Nachrichten aus der Zukunft in Abhängigkeit davon, wie wir aus der Krise herauskommen. Seien wir optimistisch!

Wann?

1. Dezember 2021

3. Mai 2024

1. Januar 2030

4. Juli 2023

\section{Nachricht}

Die Idee, globale Krisen nur durch globale Kooperation lösen zu können, hat sich weltweit durchgesetzt, sogar in Ungarn, Polen, China, Russland und (ganz zuletzt) in den USA. Der US-amerikanische Präsident tritt der Nato und den Vereinten Nationen am Nationalfeiertag der USA mit großer Zeremonie bei. Der Wiedereintritt war notwendig, weil sein Vorgänger im September 2020 beide Institutionen verlassen hatte, um den verlorenen Wahlkampf wegen der mittlerweile 2 Millionen Covid-19-Toten noch einmal herumzureißen, und zu zeigen, dass nur er für die Amerikaner (und für sonst niemanden) einsteht. Es hatte nichts genutzt: Sein Motto "America first" war ihm auf die Füße gefallen, denn es bezeichnete damals seit Monaten vor allem die Position der USA auf der Rangliste der Staaten mit den meisten Corona-Opfern.

Unter dem Motto „Influenza statt Influencer“ vereinigen sich 15-jährige Mädchen, die Virologie studieren wollen, weil man damit erstens berühmter wird als mit Mode und Schminke und zweitens die Sache um Welten spannender ist.

Im vergangenen Monat hatte eine Virus-Pandemie mit dem Virus SARS-CoV-3 die Welt in Atem gehalten. Wegen offener Grenzen hatte es sich seit dem ersten Advent 2029 wie ein Lauffeuer innerhalb von 2 Wochen über den gesamten Erdball verbreitet. Nach einer weltweit konzertierten Aktion von Tests und Quarantäne aller Infizierten und Kontaktpersonen sowie einem seit Weihnachten in allen Ländern gestarteten Impfprogramm erklärt der Chef der WHO die Pandemie heute für beendet. Er beklagt weltweit 17 Tote.

se zum Virus und der Krankheit in wenigen Wochen veraltet wäre. Anders ist es mit dem Wissen über unser Erleben, Fühlen, Bewerten, Entscheiden und Handeln - als Einzelne, als Gruppe und als Gesellschaft. Jede Krise bringt das Beste und das Schlechteste aus den Menschen heraus, je nach Mensch eben: Egoistische Hamsterkäufe und nachbarschaftliche Hilfe beim Einkaufen, Denunziantentum und Demonstration von Solidarität, Einsamkeit und Dichtestress, das Bedürfnis nach menschlichem Kontakt (vielleicht sogar von Schülern nach den Lehrern) und vermehrte Aggressivität. Es darf nicht geschehen, dass wir wegen der Pandemie unsere Werte über Bord werfen. Die Freiheitseinschränkungen werden wieder aufgehoben werden, nach und nach, begleitet von zusätzlichen Maßnahmen wie Schutzmasken und Tracking-Apps, die mit der europäischen Datenschutzgrundverordnung vereinbar sind. Das Leben muss nicht in jeder Hinsicht (Ressourcenverbraucht, Flugreisen, sinnlose Events und vor allem immer schneller, höher, weiter) wieder genau so werden, wie es vorher war! Vielleicht wird es bewusster, nachhaltiger und solidarischer. 
[1] Adolph C, Amano K, Bang-Jensen B et al. Pandemic Politics: Timing State-Level Social Distancing Responses to COVID-19. 28.3.2020 University of Washington, Seattle, USA https://faculty.washington.edu/ cadolph/papers/AABFW2020.pdf; abgerufen am 5.4.2020

[2] Andersen KG, Rambaut A, Lipkin WI et al. The proximal origin of SARSCoV-2. Nature Medicine 2020, doi.org/10.1038/s41591-020-0820-9

[3] Anderson J. Social distancing isn't the right language for what Covid-19 asks of us. Quarzt, 3.4.2020 https://qz.com/1830347/social-distancing-isnt-the-right-language-for-what-covid-19-asks-of-us/; abgerufen am 5.4.2020

[4] Anonymus. Corona-Regeln: Wenige Verstöße - viele Anzeigen. NDR 1 Radio MV, 5.4.2020, 10:00 Uhr https://www.ndr.de/nachrichten/ mecklenburgvorpommern/Corona-Regeln-Wenige-VerstoessevieleAnzeigen,coronavirus1168.html; Abgerufen am 5.4.2020

[5] Anonymus. Coronavirus Live Updates: Pelosi Accuses Trump of ,Deadly' Denial; New York Mayor Says Medical Supplies Will Run Out in a Week. The New York Times 29.3.2020 https://nyti.ms/2xtnnwm; abgerufen am 29.3.2020

[6] ARD-Deutschlandtrend. Nur jeder Zweite in Corona-Sorge. 20.3.2020. www.bild.de/politik/inland/politik-inland/ard-deutschland-jeder-zweite-deutsche-in-corona-sorge-69505740.bild.html; abgerufen am 22.3.2020

[7] Armus T, Taylor A. Trump projects at least 100,000 coronavirus deaths in U.S. as hopes of swift recovery fade. The Washington Post 1.4.2020 https://www.washingtonpost.com/world/2020/04/01/coronavirus-latest-news/; abgerufen am 1.4.2020

[8] Brooks SK, Webster RK, Smith LE et al. The psychological impact of quarantine and how to reduce it: rapid review of the evidence. Lancet 2020; 395: 912-920

[9] Ferguson NM, Laydon D et al. Impact of non-pharmaceutical interventions (NPIs) to reduce COVID-19 mortality and healthcare demand. WHO Collaborating Centre for Infectious Disease Modelling; MRC Centre for Global Infectious Disease Analysis; Abdul Latif Jameel Institute for Disease and Emergency Analytics; Imperial College London 2020. doi.org/10.25561/77482; abgerufen am 16.3.2020

[10] Fox J, Mayes BR, Schaul K et al. 2,147 people have die from coronavirus in the US. The Washington Post 27.3.2020 https://www.washingtonpost.com/graphics/2020/national/coronavi...th\&utm_medium=email\&utm_source=newsletter\&wpisrc=nl_tyh\&wpmk=1; abgerufen am 29.3.2020

[11] Hatchett R], Mecher CE, Lipsitch M. Public health interventions and epidemic intensity during the 1918 influenza pandemic. PNAS 2007; 104: $7582-7587$

[12] Lawton G. Analysis suggests UK still not doing enough to prevent covid-19 deaths. New Scientist 17.3.2020. www.newscientist.com/ article/2237755-analysis-suggests-uk-still-not-doing-enough-to-prevent-covid-19-deaths/; abgerufen am 17.3.2020

[13] Le Page M. Why we still don't know what the death rate is for covid-19. New Scientist, 3.4.2020 https://www.newscientist.com/ article/2239497-why-we-still-dont-know-what-the-death-rate-is-forcovid-19/\#ixzz6lkd86beD; abgerufen am 5.4.2020

[14] LeungNHL, Chu DKW, Shiu EYC et al. Respiratory virus shedding in exhaled breath and efficacy of face masks. Nature Medicine2020; https://www.nature.com/articles/s41591-020-0843-2.pdf

[15] Love K. Boomer Remover. CounterPunch. org. www.counterpunch.org; abgerufen am 27.3.2020
[16] Lu D. The hunt for patient zero: Where did the coronavirus outbreak start? New Scientist, 1.4.2020 https://www.newscientist.com/ article/mg24532764-000-the-hunt-for-patient-zero-where-did-thecoronavirus-outbreak-start/?utm_campaign=onesignal\&utm_medium=alert\&utm_source=editorial; abgerufen am 1.4.2020

[17] Parpia AS, Ndeffo-Mbah ML, Wenzel NS et al. Effects of Response to 2014-2015 Ebola Outbreak on Deaths from Malaria, HIV/AIDS, and Tuberculosis, West Africa. Emerging Infectious Diseases 2016; 22 doi: 10.3201/eid2203.150977; abgerufen am 3.4.2020

[18] Peters R, Wolf C, Morcinek M. Coronavirus tödlich verharmlost. Trumps Versagen in Zitaten und Zahlen. n-tv, 4.4.2020. www.n-tv.de/ infografik/Trumps-Versagen-in-Zitaten-und-Zahlen-article21692579. html; abgerufen am 5.4.2020

[19] Science News Staff. United States strains to act as cases set record. Conflicting messages, lack of coordination plague chaotic efforts to slow coronavirus. Science 2020; 368: 17-18

[20] Spitzer M. Kant on Schizophrenia. In: Spitzer M, Maher BA (Hrsg): Philosophy and Psychopathology. Papers presented at a meeting at the Dept. of Psychology, Harvard University 1989, Berlin: Springer, 1990

[21] Spitzer M. Pandemie! Und wer geht hin? Nervenheilkunde 2009; 28 : 505-508

[22] Spitzer M. Einsamkeit. München: Droemer 2018

[23] Tversky A, Kahneman D. Judgment under uncertainty: Heuristics and biases. Science 2014; 185: 1124-1131

[24] Vaughan. Greta Thunberg says she may have had covid-19 and has self-isolated. New Scientist 24.3.2020 https://www.newscientist. com/article/2238364-greta-thunberg-says-she-may-have-had-covid19-and-has-self-isolated/?utm_campaign=onesignal\&utm_medium=alert\&utm_source=editorial; abgerufen am 24.3.2020

[25] Verity R, Okell LC, Dorigatti I et al. Estimates of the severity of coronavirus disease 2019: a model-based analysis. Lancet 2020; March 30. doi.org/10.1016/S1473-3099(20)30243-7

[26] Wang $H$, Shao J, Luo $X$ et al. Wildlife consumption ban is insufficient. Science 2020; 367: 1435

[27] Yang N, Liu P, Li W et al. Permanently ban wildlife consumption. Science 2020; 367: 1434-1435

[28] Yuan J, Morgan R. As toll grows, a new worry: Morgue capacity and bodies as sources of infection. The Washington Post 28.3.2020 https:// www.washingtonpost.com/national/coronavirus-morgue-aut...h\&utm_ medium=email\&utm_source=newsletter\&wpisrc=nl_tyh\&wpmk=1; abgerufen am 29.3.2020

[29] Zhang T, Wu Q, Zhang Z. Probable Pangolin Origin of SARS-CoV-2 Associated with the COVID-19 Outbreak, Current Biology 2020. doi. org/10.1016/j.cub.2020.03.022; abgerufen am 1.4.2020

[30] Pueyo T. Coronavirus: Why You Must Act Now. Politicians, Community Leaders and Business Leaders: What Should You Do and When? https://medium.com/@tomaspueyo/coronavirus-act-today-or-people-will-die-f4d3d9cd99ca; abgerufen am 20.3.2020

[31] Kaiser J. „Every day is a new surprise.“ Inside the effort to produce the world's most popular coronavirus tracker. Science online 6.4.2020 www.sciencemag.org/news/2020/04/every-day-new-surprise-inside-effort-produce-world-s-most-popular-coronavirus-tracker; abgerufen am 7.4.2020 